\title{
A path planning approach of 3D manipulators using zenithal gnomic projection and polar piecewise interpolation
}

\author{
Mihai Dupac* \\ Talbot Campus, Fern Barrow, Poole, Dorset BH12 5BB \\ Department of Design and Engineering, Bournemouth University, ${ }^{1, *}$
}

\begin{abstract}
In this paper the mathematical modeling and trajectory planning of a 3D rotating manipulator composed of a rotating-prismatic joint and multiple rigid links is considered. Possible trajectories of the end-effector of the manipulator following a sequence of $3 \mathrm{D}$ target points under the action of two external driving torques and an axial force - are modelled using zenithal gnomic projections and polar piecewise interpolants expressed as polynomial Hermite-type functions. Due to the geometry of the manipulator, the time dependent generalized coordinates are associated with the spherical coordinates named the radial distance related to the manipulator length, and the polar and azimuthal angles describing the left and right, and respectively up and down motion of the manipulator. The polar trajectories (left and right motion) of the end-effector are generated using a inverse geometric transformation applied to the polar piecewise interpolants that approximate the gnomic projective trajectory of the $3 \mathrm{D}$ via-points. The gnomic via-points - located on a projective plane situated on the northern hemisphere - are seen from the manipulator base location which represents the center of rotation of the extensible manipulator. The related azimuthal trajectory (up and down motion) is generated by polar piecewise interpolants on the
\end{abstract}

\footnotetext{
*Corresponding author: Mihai Dupac

Email address: mdupac@bournemouth.ac.uk (Department of Design and Engineering, Bournemouth University)
}

Preprint submitted to Journal of LATE $_{E} X$ Templates

December 30, 2017 
azimuthal angles. Smoothness of the polygonal trajectory is obtained through the use of piecewise interpolants with continuous derivatives, while the kinematics and dynamics implementation of the model is well suited to computer implementation (easy calculation of kinematics variables) and simulation. To verify the approach and validate the model a numerical example - implemented in Matlab - is presented and the results are discussed.

Keywords: Kinematics, dynamics, piecewise interpolation, trajectory planning 2010 MSC: 65D05, 70B15

\section{Introduction}

The use of numerical methods in solving engineering problems [5, 10] has increased considerably in recent years. The development of such numerical methods including numerical integration and numerical solutions of PDE generated advancement in all fields of engineering: automatic production lines and industrial robots represent some explicit examples. Extending industrial robot capabilities along production lines represents a critical key element to reduce production costs and improve productivity in industry. The ability to control the motion along a desired trajectory [12] is imperative, especially when some features required at high operating speeds - such as kinematics and dynamics constraints [15], execution time [3, 6], smooth (hereby reducing wear on the robot) trajectory generation [10] and jerk [7 - are considered.

Implicit and explicit methods [22] are considered for the design, simulation and control of industrial robots. The implicit methods are based on a potential field while the explicit methods [22] can be split in discrete and continuous methods called kinematic or dynamic motion planning [10, 26, 22]. Both the kinematics and dynamics models are indispensable for the simulation and control of novel industrial robots. Therefore, adaptive control schemes for realistic control of robotic arms should be considered [2] using trajectory planning and manipulation [26] strategies. Such trajectory planning [24] should diminish the 
manipulator induced vibrations, execution time and wear.

The increased requirements of new manipulators [1] demand rigorous trajectory planning studies which by the means of adequate control should generate smooth (high order of continuity) trajectories. The trajectory of a mobile manipulator with flexible links that allows carrying a maximum load between two specified positions was considered and formulated as a trajectory optimization problem in [11. An optimal control approach for finding the maximum carrying load capacity based on an indirect (explicit) solution have been addressed in [19]. The corresponding joint optimal path planning of end-effectors subject to actuator torque limits is presented and solved in [20] using inverse kinematic, associated Hamiltonian function and Pontryagins minimum principle. A new method of a hierarchical optimal control through system decoupling have been introduced in [21] for maximum allowable load calculation and path planning.

The trajectories used in path planning can have different representations, among them, piecewise interpolating curves [16, parametric and/or geometric continuous splines [28, 13, or uniform cubic B-spline with parametric and geometric continuity, have been usually considered [17, 14, 16, 17]. The interpolation of smooth curves for generating orientation trajectories with minimal angular acceleration is presented in [17. A new interpolation methodology for the path-planning of an industrial robot and a set of prescribed kinematical requirements is presented in [27]. Algebraic-trigonometric Hermite polynomial curves that are very practical in generating smooth and continuous manipulator motion are considered in [29]. Smooth $\left(C^{1}\right)$ univariate cubic $\left(L_{1}\right)$ interpolating splines in polar and Cartesian coordinates are presented in [23, and cross and circum-polar continuity based on the interpolation on a non-uniform latitudelongitude are discussed in [14.

In this paper the modelling and simulation of $3 \mathrm{D}$ trajectory of the endeffector of a rotating extensible manipulator arm composed of a rotating-prismatic joint and multiple rigid links is considered. The geometric path of the manipulator is generated using a inverse geometric transformation applied to the polar planar curves that approximate the gnomic projective trajectory of the $3 \mathrm{D}$ via 
points and the related azimuthal trajectories on the azimuthal angles. Both the polar and azimuthal trajectories are generated by polar piecewise interpolants expressed as polynomial Hermite-type functions. To verify the proposed approach, a numerical simulation is performed and the results are discussed.

\section{Mathematical modelling of the end-effector trajectory}

Manipulator model. The rotating extensible manipulator arm is composed of a rotating column base (link 1), a rotating slider joint (link 2) and a sliding link (link 3) as shown in Fig. 11. The link 1 of the manipulator can rotate at $O_{0}$ - in the fixed Cartesian reference frame $O_{0} x_{0} y_{0} z_{0}$ (named reference frame (0)) about the vertical axis $O_{0} z_{0}$ as shown in Fig. 2. The fixed reference frame (0) is defined by the unit vectors $\mathbf{i}_{0}, \mathbf{j}_{0}, \mathbf{k}_{0}$. A mobile reference frame $O_{1} x_{1} y_{1} z_{1}$ (reference frame (1) attached to link 1) shown in Fig. 2 is also attached to the rotating link (1). The mobile reference frame $O_{1} x_{1} y_{1} z_{1}$ is defined by $O_{0}=O_{1}$, $O_{0} z_{0}=O_{1} z_{1}$, the unit vector $\mathbf{k}_{1}=\mathbf{k}_{0}$, and the unit vectors $\mathbf{i}_{1}, \mathbf{j}_{1}$ that can rotate about $k_{0}$. Link 1 is connected to link 2 through pin joints $B$ and $B_{0}$ shown in Fig. 3. The link 2 (Fig. 3) contains the slider joint which is rigidly attached to it. The link 2 rotates relative to 1 about an axis passing through $B_{0}$, and $B_{1}$. A fixed Cartesian reference frame $\mathrm{O}_{02} x_{02} y_{02} z_{02}$ (named reference frame (02) parallel to $O_{0} x_{0} y_{0} z_{0}$ (reference frame (0)) is defined at $O_{02}=O_{2}$ by the unit vectors $\mathbf{i}_{02}, \mathbf{j}_{02}, \mathbf{k}_{02}$. A mobile reference frame (2) of unit vectors $\mathbf{i}_{2}, \mathbf{j}_{2}, \mathbf{k}_{2}$ is also attached to link 2, as shown in Fig. 3. The sliding link (link 3) which is interconnected with the slider (link 2) can slide in and out of the slider. The length of link 1 is $l_{1}$, the length of the link 2 is $l_{2}$ and the length of the link 3 is $l_{3}$.

Trajectory generation. The geometric path of the end-effector (Fig. 1 is specified in the fixed Cartesian reference frame $O_{02} x_{02} y_{02} z_{02}$ by piecewise interpolants between the via-points $P_{1}, P_{2}, \ldots, P_{k}, \ldots, P_{n}$ (Fig. 11). The geometric path of the end-effector is given in spherical coordinates by the position vector

$$
\mathbf{r}_{P_{i_{k}}}=l_{P_{i_{k}}} \sin \varphi_{P_{i_{k}}} \cos \theta_{P_{i_{k}}} \mathbf{i}_{02}+l_{P_{i_{k}}} \sin \varphi_{P_{i_{k}}} \sin \theta_{P_{i_{k}}} \mathbf{j}_{02}+l_{P_{i_{k}}} \cos \varphi_{P_{i_{k}}} \mathbf{k}_{02}
$$




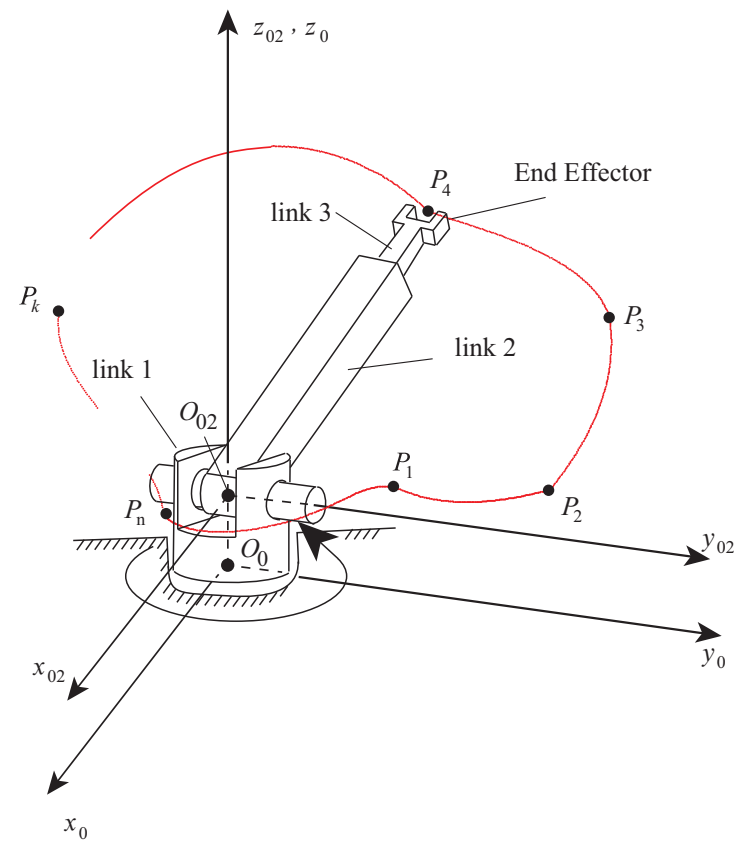

Figure 1: Rotating extensible manipulator model

where $P_{i_{k}}$ represents the interpolating points along the piecewise curve defined by the via-points $P_{i}$ and $P_{i+1}, l_{P_{i_{k}}}=d\left(O_{02}, P_{i_{k}}\right)$ is the radial distance/radius from the point $O_{02}$ of the fixed reference frame $O_{02} x_{02} y_{02} z_{02}$ to the point $P_{i_{k}}$, $l_{P_{i}}=d\left(O_{02}, P_{i}\right)$ is the distance from $O_{02}$ to the point $P_{i_{k}}, z_{P_{i_{k}}}, \theta_{P_{i_{k}}}$ is the polar angle given in an anticlockwise direction, and $\varphi_{P_{i_{k}}}$ is the azimuthal angle.

The end-effector of the manipulator arm should move along the via-points $P_{1}, P_{2}, \ldots, P_{k}, \ldots, P_{n}$ set by the user (Fig. 11). To generate the geometric path e.g., to interpolate between the data, one can consider the perspective projections of the via-points - viewed from the origin $\mathrm{O}_{02}$ (which represents the centre of projection) of the reference frame $O_{02} x_{02} y_{02} z_{02}$ - to a plane $N$ perpendicular to the axis $O_{02} 0 z_{02}$ and located at the distance $l_{M a x}=\max \left\{l_{P_{i}}\right\}_{i=\overline{0, N_{i}}}$ from the origin $\mathrm{O}_{02}$ (and tangent to the sphere of radius $l_{\text {Max }}$ centred in $O_{02}$ ) as shown in Fig. 4. Due to the geometry of the manipulator arm, polar zenithal gnomic projections are associated with the perspective projections denoted by 
$P_{i}^{\prime}\left(r_{P_{i}^{\prime}}, \theta_{i}, \varphi_{i}\right)=P_{i}^{\prime}\left(X_{P_{i}^{\prime}}, Y_{P_{i}^{\prime}}, Z_{P_{i}^{\prime}}\right)$ and calculated using

$$
\left\{\begin{array}{l}
\frac{X_{P_{i}^{\prime}}-x_{O_{02}}}{x_{P_{i}}-x_{O_{02}}}=\frac{Y_{P_{i}^{\prime}}-y_{O_{02}}}{y_{P_{i}}-y_{O_{02}}}=\frac{Z_{P_{i}^{\prime}}-z_{O_{02}}}{z_{P_{i}}-z_{O_{02}}} \\
Z_{P_{i}^{\prime}}=l_{M a x}=\max \left\{l_{P_{i}^{\prime}}\right\}_{i=\overline{0, N_{i}}}
\end{array}\right.
$$

or equivalent by

$$
\left\{\begin{array}{c}
X_{P_{i}^{\prime}}=x_{O_{02}}+\frac{x_{P_{i}}-x_{O_{02}}}{z_{P_{i}}-z_{O_{02}}}\left(l_{M a x}-z_{O_{02}}\right) \\
Y_{P_{i}^{\prime}}=y_{O_{02}}+\frac{y_{P_{i}}-y_{O_{02}}}{z_{P_{i}}-z_{O_{02}}}\left(l_{M a x}-z_{O_{02}}\right)
\end{array}\right.
$$

that is the intersection of the lines $O_{02} P_{i}$ passing by $O_{02}$ and $P_{i}$ with the plane $N$. The associated radii $R_{P_{i}^{\prime}}=d\left(O^{\prime}, P_{i}^{\prime}\right)$ located in the projective plane $N$ are calculated from Eq. (3) as

$$
\begin{aligned}
R_{P_{i}^{\prime}} & =d\left(O^{\prime}, P_{i}^{\prime}\right)=\sqrt{\left(X_{P_{i}^{\prime}}\right)^{2}+\left(Y_{P_{i}^{\prime}}\right)^{2}} \\
& =\sqrt{\begin{array}{l}
\left(x_{O_{02}}+\frac{x_{P_{i}}-x_{O_{02}}}{z_{P_{i}}-z_{O_{02}}}\left(l_{M a x}-z_{O_{02}}\right)\right)^{2} \\
+\left(y_{O_{02}}+\frac{y_{P_{i}}-y_{O_{02}}}{z_{P_{i}}-z_{O_{02}}}\left(l_{M a x}-z_{O_{02}}\right)\right)^{2}
\end{array}}
\end{aligned}
$$

Since in a gnomic projection the projection center is the center of the reference frame, that is, $x_{O_{02}}=0, y_{O_{02}}=0$, and $z_{O_{02}}=0$, Eq. (4) can be written as

$$
\begin{aligned}
& R_{P_{i}^{\prime}} \stackrel{\text { Gnomic }}{=} d\left(O^{\prime}, P_{i}^{\prime}\right)=\sqrt{\left(X_{P_{i}^{\prime}}\right)^{2}+\left(Y_{P_{i}^{\prime}}\right)^{2}} \\
&=\sqrt{\left(\frac{x_{P_{i}}}{z_{P_{i}}}\left(l_{\text {Max }}\right)\right)^{2}+\left(\frac{y_{P_{i}}}{z_{P_{i}}}\left(l_{\text {Max }}\right)\right)^{2}} \\
&=\frac{\rho_{P_{i}}}{z_{P_{i}}} l_{\text {Max }}
\end{aligned}
$$

The gnomic projections $P_{i}^{\prime}$ and $P_{i+1}^{\prime}$ of the $3 \mathrm{D}$ via points $P_{i}$ and $P_{i+1}$ on the projective plane $N$ are shown in Fig. 4. To interpolate between the gnomic via-points $P_{i}^{\prime}, i=\overline{0, N_{i}}$ (Fig. 4 a piecewise polar interpolation was considered. For each interval $\left[\theta_{P_{i}^{\prime}}, \theta_{P_{i+1}^{\prime}}\right]=\left[\theta_{i}, \theta_{i+1}\right]_{i=0, N_{i}-1}$ and associated radii $R_{P_{i}^{\prime}}$ and $R_{P_{i+1}^{\prime}}$ of the consecutive points $P_{i}^{\prime}$ and $P_{i+1}^{\prime}$, a piecewise polar interpolant that approximates trajectory in the projective plane $N$ (Fig. 4) can be expressed as 


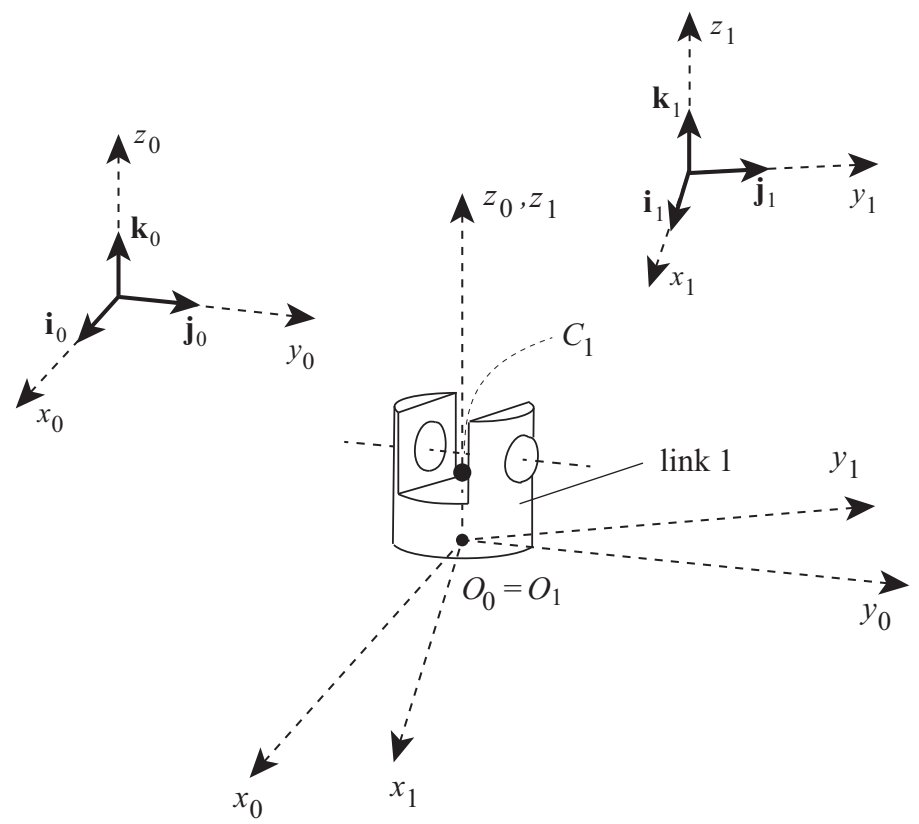

Figure 2: Schematic representation of the link 1 of the manipulator consisting of three links 1,2 , and 3 .

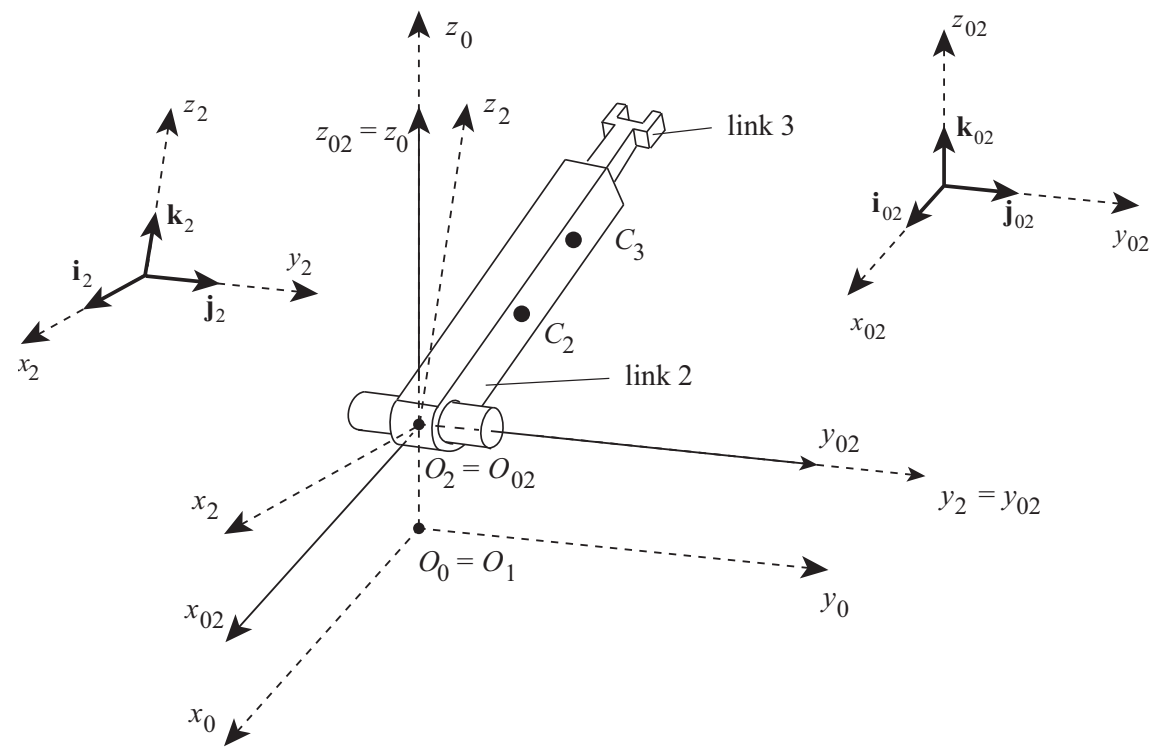

Figure 3: Schematic representation of the links 2 and 3 of the manipulator consisting consisting of three links 1,2 , and 3 . 


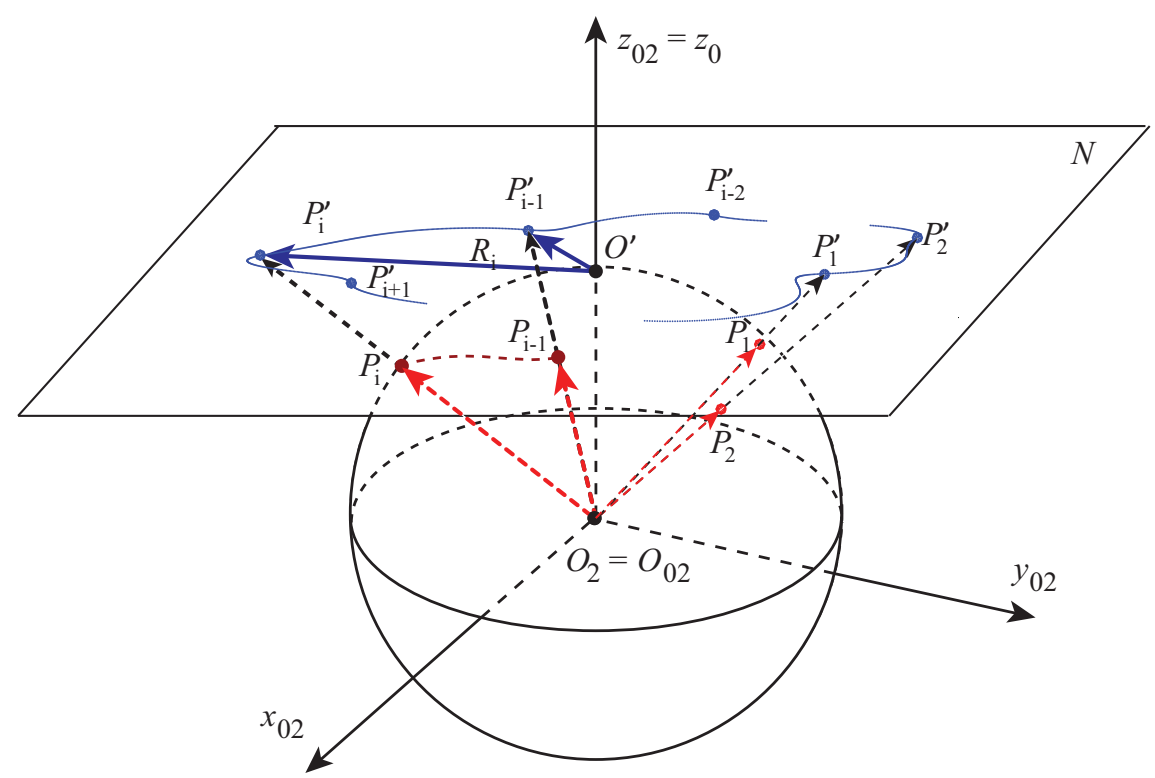

Figure 4: Perspective projections of the via-points $P_{i}$, and associated piecewise interpolation

a Hermite-type function [8, 9, 16, 23] defined by

$$
R(\theta)=\sum_{k=0}^{q} c_{k}^{i}\left(\theta-\theta_{P_{i}^{\prime}}\right)^{k}=\sum_{k=0}^{q} c_{k}^{i}\left(\theta-\theta_{i}\right)^{k}
$$

where $q$ is the order of the polynomial to be considered (the order is 3 in this approximation $), c_{0}^{i}=R_{P_{i}^{\prime}}, c_{1}^{i}=\dot{R}_{P_{i}^{\prime}}, c_{2}^{i}=\frac{1}{h_{P_{i}^{\prime}}}\left[\left(2 \dot{R}_{P_{i}^{\prime}}+\dot{R}_{P_{i+1}^{\prime}}\right)+3 \Delta R_{P_{i}^{\prime}}\right]$, $c_{3}^{i}=\frac{1}{h_{P_{i}^{\prime}}^{2}}\left[\dot{R}_{P_{i}^{\prime}}+\dot{R}_{P_{i+1}^{\prime}}-2 \Delta R_{P_{i}^{\prime}}\right], R_{P_{i}^{\prime}}=R\left(\theta_{P_{i}^{\prime}}\right), R_{P_{i+1}^{\prime}}=R\left(\theta_{P_{i+1}^{\prime}}\right), h_{P_{i}^{\prime}}=$ $\theta_{P_{i+1}^{\prime}}-\theta_{P_{i}^{\prime}}, \Delta y_{P_{i}^{\prime}}=\frac{R_{P_{i+1}^{\prime}}-R_{P_{i}^{\prime}}}{h_{P_{i}^{\prime}}}$, and where $\theta_{P_{i}^{\prime}}=\theta_{i}$ and $\theta_{P_{i+1}^{\prime}}=\theta_{i+1}$. The derivatives at the endpoints $P_{i}^{\prime}$ and $P_{i+1}^{\prime}$ are calculated as $\dot{R}\left(\theta_{P_{i}^{\prime}}\right)=\frac{d R\left(\theta_{P_{i}^{\prime}}\right)}{d \theta}=$ $\dot{R}_{P_{i}^{\prime}}$ and $\dot{R}\left(\theta_{P_{i+1}^{\prime}}\right)=\frac{d R\left(\theta_{P_{i+1}^{\prime}}\right)}{d \theta}=\dot{R}_{P_{i+1}^{\prime}}$ respectively.

The 3D trajectories of the end-effector derived from Eq. (5) can be calculated using the geometric transformation below

$$
x=\frac{1}{l_{\text {Max }}} R(\theta) l(\varphi) \cos \theta, \quad y=\frac{1}{l_{\text {Max }}} R(\theta) l(\varphi) \sin \theta, \quad z=z(\theta)
$$

The length $l(\varphi)$ which relates trajectory height with the arm length is com- 
puted using a piecewise polar interpolation related to the azimuthal angles. For each interval $\left[\varphi_{P_{i}}, \varphi_{P_{i+1}}\right]=\left[\varphi_{i}, \varphi_{i+1}\right]_{i=\overline{0, N_{i}-1}}$ and associated radii length $l_{P_{i}}$ and $l_{P_{i+1}}$ of the consecutive points $P_{i}$ and $P_{i+1}$, a piecewise polar interpolant that approximates the trajectory along the polar angles can be expressed as a Hermite-type function [8, 9, 16, 23] defined by

$$
l(\varphi)=\sum_{k=0}^{q} b_{k}^{i}\left(\varphi-\varphi_{P_{i}}\right)^{k}=\sum_{k=0}^{q} b_{k}^{i}\left(\varphi-\varphi_{i}\right)^{k}
$$

where $q$ is the order of the polynomial to be considered (the order is 3 in this approximation), $b_{0}^{i}=l_{P_{i}}, b_{1}^{i}=i_{P_{i}}, b_{2}^{i}=\frac{1}{h_{P_{i}}}\left[\left(2 \dot{l}_{P_{i}}+\dot{l}_{P_{i+1}}\right)+3 \Delta l_{P_{i}}\right], b_{3}^{i}=$ $\frac{1}{h_{P_{i}}^{2}}\left[i_{P_{i}}+i_{P_{i+1}}-2 \Delta l_{P_{i}}\right], l_{P_{i}}=l\left(\varphi_{P_{i}}\right), l_{P_{i+1}}=l\left(\varphi_{P_{i+1}}\right), h_{P_{i}}=\varphi_{P_{i+1}}-\varphi_{P_{i}}$, $\Delta y_{P_{i}}=\frac{l_{P_{i+1}}-l_{P_{i}}}{h_{P_{i}}}$, and where $\varphi_{P_{i}}=\varphi_{i}$ and $\varphi_{P_{i+1}}=\varphi_{i+1}$. The derivatives at the endpoints $P_{i}$ and $P_{i+1}$ are calculated as $i\left(\varphi_{P_{i}}\right)=\frac{d l\left(\varphi_{P_{i}}\right)}{d \varphi}=i_{P_{i}}$ and $i\left(\varphi_{P_{i+1}}\right)=\frac{d l\left(\varphi_{P_{i+1}}\right)}{d \varphi}=i_{P_{i+1}}$ respectively. The $3 \mathrm{D}$ trajectories of the endeffector can now be calculated using the geometric transformation below

$$
x=\frac{R(\theta) l(\varphi) \sin \varphi \cos \theta}{l_{\text {Max }}}, \quad y=\frac{R(\theta) l(\varphi) \sin \varphi \sin \theta}{l_{\text {Max }}}, \quad z=r(\varphi) \cos \varphi
$$

Path Planning. The geometric path of the end-effector considered in Eq. (1) can be parameterized using the unit tangent, unit normal, and the unit binormal vector $\mathbf{e}_{\theta}, \mathbf{e}_{r}$ and $\mathbf{e}_{\varphi}$ expressed as $\mathbf{e}_{r}=\sin \theta \cos \varphi \mathbf{i}_{0}+\sin \theta \sin \varphi \mathbf{j}_{0}+\cos \theta \mathbf{k}_{0}$, $\mathbf{e}_{\theta}=\cos \theta \cos \varphi \mathbf{i}_{0}+\cos \theta \sin \varphi \mathbf{j}_{0}-\sin \theta \mathbf{k}_{0}$, and $\mathbf{e}_{\varphi}=-\sin \theta \mathbf{i}_{0}+\cos \varphi \mathbf{j}_{0}$ by

$$
\begin{aligned}
\mathbf{r}= & l \mathbf{e}_{r} \\
\mathbf{v}= & i \mathbf{e}_{r}+l \dot{\theta} \mathbf{e}_{\theta}+l \dot{\varphi} \sin \theta \mathbf{e}_{\varphi} \\
\mathbf{a}= & \left(\ddot{l}-l \dot{\theta}^{2}-l \dot{\varphi}^{2} \sin ^{2} \theta\right) \mathbf{e}_{r} \\
& +\left(l \ddot{\theta}+2 i \dot{\theta}-l \dot{\varphi}^{2} \sin \theta \cos \theta\right) \mathbf{e}_{\theta} \\
& +(l \ddot{\varphi} \sin \theta+2 \dot{l} \dot{\varphi} \sin \theta+2 l \dot{\theta} \dot{\varphi} \cos \theta) \mathbf{e}_{\varphi}
\end{aligned}
$$


The first and second derivative of $R=R(\theta)$ and $l=l(\varphi)$ in Eq. (6) and Eq. (8) can be calculated using

$$
\begin{aligned}
\dot{R}(\theta) & =\dot{\theta} \sum_{k=0}^{3} c_{k}^{i} k\left(\theta-\theta_{i}\right)^{k-1} \\
\ddot{R}(\theta) & =\ddot{\theta} \sum_{k=1}^{3} c_{k}^{i} k\left(\theta-\theta_{i}\right)^{k-1}+\dot{\theta}^{2} \sum_{k=2}^{3} c_{k}^{i}(k-1) k\left(\theta-\theta_{i}\right)^{k-2} \\
\dot{l}(\varphi) & =\dot{\varphi} \sum_{k=0}^{3} b_{k}^{i} k\left(\varphi-\varphi_{i}\right)^{k-1} \\
\ddot{l}(\varphi) & =\ddot{\varphi} \sum_{k=1}^{3} b_{k}^{i} k\left(\varphi-\varphi_{i}\right)^{k-1}+\dot{\varphi}^{2} \sum_{k=2}^{3} b_{k}^{i}(k-1) k\left(\varphi-\varphi_{i}\right)^{k-2}
\end{aligned}
$$

The position vector of the end-effector can be expressed in terms of Eq. 6], Eq. (8) and Eq. (9) by

$$
\begin{aligned}
\mathbf{r}_{x} & =\frac{1}{l_{\text {Max }}} R(\theta) l(\varphi) \sin \varphi \cos \theta \\
& =\frac{1}{l_{\text {Max }}} \sum_{k=0}^{q} c_{k}^{i}\left(\theta-\theta_{i}\right)^{k} \sum_{k=0}^{q} b_{k}^{i}\left(\varphi-\varphi_{i}\right)^{k} \sin \varphi \cos \theta \\
\mathbf{r}_{y} & =\frac{1}{l_{\text {Max }}} R(\theta) l(\varphi) \sin \varphi \sin \theta \\
& =\frac{1}{l_{\text {Max }}} \sum_{k=0}^{q} c_{k}^{i}\left(\theta-\theta_{i}\right)^{k} \sum_{k=0}^{q} b_{k}^{i}\left(\varphi-\varphi_{i}\right)^{k} \sin \varphi \sin \theta \\
\mathbf{r}_{z} & =l(\varphi) \cos \varphi \\
& =\sum_{k=0}^{q} b_{k}^{i}\left(\varphi-\varphi_{i}\right)^{k} \cos \varphi
\end{aligned}
$$

The end-effector velocity vector can now be calculated using Eq. (6), Eq. (8) and Eq. (9) by

$$
\begin{aligned}
\mathbf{v}_{x}= & \frac{1}{l_{\text {Max }}}[\dot{R}(\theta) l(\varphi) \sin \varphi \cos \theta+R(\theta) \dot{l}(\varphi) \sin \varphi \cos \theta \\
& +R(\theta) l(\varphi) \dot{\varphi} \cos \varphi \cos \theta-R(\theta) l(\varphi) \dot{\theta} \sin \varphi \sin \theta] \\
\mathbf{v}_{y}= & \frac{1}{l_{\text {Max }}}[\dot{R}(\theta) l(\varphi) \sin \varphi \sin \theta+R(\theta) \dot{l}(\varphi) \sin \varphi \sin \theta \\
& +R(\theta) l(\varphi) \dot{\varphi} \cos \varphi \sin \theta+R(\theta) l(\varphi) \dot{\theta} \sin \varphi \cos \theta] \\
\mathbf{v}_{z}= & i(\varphi) \cos \varphi-l(\varphi) \dot{\varphi} \sin \varphi
\end{aligned}
$$


or equivalent

$$
\begin{aligned}
& \mathbf{v}_{x}=\frac{1}{l_{M a x}}\left[\dot{\theta} \sum_{k=0}^{3} c_{k}^{i} k\left(\theta-\theta_{i}\right)^{k-1} \sum_{k=0}^{q} b_{k}^{i}\left(\varphi-\varphi_{i}\right)^{k} \sin \varphi \cos \theta\right. \\
& +\dot{\varphi} \sum_{k=0}^{q} c_{k}^{i}\left(\theta-\theta_{i}\right)^{k} \sum_{k=0}^{3} b_{k}^{i} k\left(\varphi-\varphi_{i}\right)^{k-1} \sin \varphi \cos \theta \\
& +\dot{\varphi} \sum_{k=0}^{q} c_{k}^{i}\left(\theta-\theta_{i}\right)^{k} \sum_{k=0}^{q} b_{k}^{i}\left(\varphi-\varphi_{i}\right)^{k} \cos \varphi \cos \theta \\
& \left.-\dot{\theta} \sum_{k=0}^{q} c_{k}^{i}\left(\theta-\theta_{i}\right)^{k} \sum_{k=0}^{q} b_{k}^{i}\left(\varphi-\varphi_{i}\right)^{k} \sin \varphi \sin \theta\right] \\
& \mathbf{v}_{y}=\frac{1}{l_{\text {Max }}}\left[\dot{\theta} \sum_{k=0}^{3} c_{k}^{i} k\left(\theta-\theta_{i}\right)^{k-1} \sum_{k=0}^{q} b_{k}^{i}\left(\varphi-\varphi_{i}\right)^{k} \sin \varphi \sin \theta\right. \\
& +\dot{\varphi} \sum_{k=0}^{q} c_{k}^{i}\left(\theta-\theta_{i}\right)^{k} \sum_{k=0}^{3} b_{k}^{i} k\left(\varphi-\varphi_{i}\right)^{k-1} \sin \varphi \sin \theta \\
& +\dot{\varphi} \sum_{k=0}^{q} c_{k}^{i}\left(\theta-\theta_{i}\right)^{k} \sum_{k=0}^{q} b_{k}^{i}\left(\varphi-\varphi_{i}\right)^{k} \cos \varphi \sin \theta \\
& \left.+\dot{\theta} \sum_{k=0}^{q} c_{k}^{i}\left(\theta-\theta_{i}\right)^{k} \sum_{k=0}^{q} b_{k}^{i}\left(\varphi-\varphi_{i}\right)^{k} \sin \varphi \cos \theta\right] \\
& \mathbf{v}_{z}=\dot{\varphi} \sum_{k=0}^{3} b_{k}^{i} k\left(\varphi-\varphi_{i}\right)^{k-1} \cos \varphi-\sum_{k=0}^{q} b_{k}^{i}\left(\varphi-\varphi_{i}\right)^{k} \dot{\varphi} \sin \varphi
\end{aligned}
$$

Kinematics. To characterize the instantaneous position of the manipulator, e.g., centers of mass $C_{0}, C_{1}$, and $C_{2}$, of each link (link 0, link 1 and link 2) of the manipulator, the generalized coordinates $\theta, \varphi$ and $l_{C_{3}}$ are considered. The same reference frames as before are now considered. The first generalized coordinate $\theta_{C_{1}}$ denotes the radian measure between the axes $O_{0} z_{0}$ and $O_{1} z_{1}$ described by the unit vectors $\mathbf{i}_{0}$ and $\mathbf{i}_{1}$. The second generalized coordinate $\varphi_{C_{2}}$ designates the radian measure of rotation of the angle between the axes $O_{1} z_{1}$ and $O_{2} z_{1}$ described by the frames (1) and (2). The last generalized coordinate $l_{C_{3}}$ is the distance from the point $O_{2}$ to the centre of the mass $C_{3}$ of the 3-rd link.

To describe the orientation of a coordinate frame relative to another coordinate frame the Euler angles can be considered. The unit vectors $\mathbf{i}_{1}, \mathbf{j}_{1}, \mathbf{k}_{1}$ can be expressed as functions of $\mathbf{i}_{0}, \mathbf{j}_{0}, \mathbf{k}_{0}$, while the unit vectors $\mathbf{1}_{2}, \mathbf{J}_{2}$ and $\mathbf{k}_{2}$ can be ex- 
pressed as functions of $\mathbf{i}_{1}, \mathbf{j}_{1}, \mathbf{k}_{1}$ by $\left[\mathbf{i}_{s}, \mathbf{j}_{s}, \mathbf{k}_{s}\right]^{T}=A_{s}\left[\mathbf{i}_{s-1}, \mathbf{j}_{s-1}, \mathbf{k}_{s-1}\right]^{T}, s=1,2$ where

$$
A_{1}=\left[\begin{array}{ccc}
\cos \theta & -\sin \theta & 0 \\
\sin \theta & \cos \theta & 0 \\
0 & 0 & 1
\end{array}\right], \quad A_{2}=\left[\begin{array}{ccc}
1 & 0 & 0 \\
0 & \cos \varphi & -\sin \varphi \\
0 & \sin \varphi & \cos \varphi
\end{array}\right]
$$

The angular velocities of the links 1,2 and 3 can be expressed by

$$
\begin{aligned}
\boldsymbol{\omega}_{10} & =\dot{\theta} \mathbf{k}_{1}=\dot{\theta} \mathbf{k}_{0} \\
\boldsymbol{\omega}_{21} & =\dot{\varphi} \mathbf{i}_{2}=\dot{\varphi} \mathbf{i}_{1}=\dot{\varphi} \cos \theta \mathbf{i}_{0}-\dot{\varphi} \sin \theta \mathbf{j}_{0} \\
\boldsymbol{\omega}_{20} & =\boldsymbol{\omega}_{10}+\boldsymbol{\omega}_{21}=\dot{\theta} \mathbf{k}_{1}+\dot{\varphi} \mathbf{i}_{2} \\
& =\dot{\varphi} \cos \theta \mathbf{i}_{0}-\dot{\varphi} \sin \theta \mathbf{j}_{0}+\dot{\theta} \mathbf{k}_{0} \\
\boldsymbol{\omega}_{30} & =\boldsymbol{\omega}_{20}
\end{aligned}
$$

where $\boldsymbol{\omega}_{10}, \boldsymbol{\omega}_{20}, \boldsymbol{\omega}_{30}$ are the angular velocities of links 1,2 and 3 in the fixed reference frame (0).

The angular accelerations of the links 1, 2 and 3 in the reference frame (0) are

$$
\begin{aligned}
\boldsymbol{\alpha}_{1} & =\ddot{\theta} \mathbf{k}_{1}=\ddot{\theta} \mathbf{k}_{0} \\
\boldsymbol{\alpha}_{20} & =\frac{d}{d t} \boldsymbol{\omega}_{20}=\frac{{ }^{(2)} d}{d t} \boldsymbol{\omega}_{20}+\boldsymbol{\omega}_{20} \times \boldsymbol{\omega}_{20}=\frac{{ }^{(2)} d}{d t} \boldsymbol{\omega}_{20}=\frac{{ }^{(0)} d}{d t} \boldsymbol{\omega}_{20} \\
& =\frac{{ }^{(0)} d}{d t}\left[\dot{\varphi} \cos \theta \mathbf{i}_{0}-\dot{\varphi} \sin \theta \mathbf{j}_{0}+\dot{\theta} \mathbf{k}_{0}\right] \\
& =(\ddot{\varphi} \cos \theta-\dot{\varphi} \dot{\theta} \sin \theta) \mathbf{i}_{0}-(\ddot{\varphi} \sin \theta+\dot{\varphi} \dot{\theta} \cos \theta) \mathbf{j}_{0}+\ddot{\theta} \mathbf{k}_{0} \\
\boldsymbol{\alpha}_{30} & =\boldsymbol{\alpha}_{20}
\end{aligned}
$$

where $\frac{{ }^{(2)} d}{d t}$ represents the derivative with respect to time in reference frame (2), $\frac{{ }^{(0)} d}{d t}$ represents the derivative with respect to time in reference frame (0), and link 3 has the same angular acceleration as link 2.

The position vectors of $C_{1}, C_{2} C_{3}$, i.e., the mass centers of link 1 , link 2 and 
link 3 , can be calculated using

$$
\begin{aligned}
\mathbf{r}_{C_{1}} & =\frac{l_{1}}{2} \mathbf{k}_{1}=\frac{l_{1}}{2} \mathbf{k}_{0} \\
\mathbf{r}_{C_{2}} & =l_{1} \mathbf{k}_{1}+\frac{l_{2}}{2} \mathbf{j}_{2} \\
& =\frac{l_{2}}{2} \cos \varphi \mathbf{j}_{1}-\frac{l_{2}}{2} \sin \varphi \mathbf{k}_{1}+l_{1} \mathbf{k}_{0} \\
& =\frac{l_{2}}{2} \cos \varphi \sin \theta \mathbf{i}_{0}+\frac{l_{2}}{2} \cos \varphi \cos \theta \mathbf{j}_{0}-\left(\frac{l_{2}}{2} \sin \varphi+l_{1}\right) \mathbf{k}_{0} \\
\mathbf{r}_{C_{3}} & =l_{1} \mathbf{k}_{1}+l_{C_{3}} \mathbf{j}_{2} \\
& =l_{C_{3}} \cos \varphi \sin \theta \mathbf{i}_{0}+l_{C_{3}} \cos \varphi \cos \theta \mathbf{j}_{0}-\left(l_{C_{3}} \sin \varphi+l_{1}\right) \mathbf{k}_{0}
\end{aligned}
$$

The velocity vectors of $C_{1}, C_{2}$, and $C_{3}$ with respect to the reference frame (0) are

$$
\begin{aligned}
\mathbf{v}_{C_{1}} & =\frac{d}{d t} \mathbf{r}_{C_{1}}=\dot{\mathbf{r}}_{C_{1}}=\mathbf{0} . \\
\mathbf{v}_{C_{2}} & =\frac{d}{d t} \mathbf{r}_{C_{2}} \\
& =\frac{d}{d t}\left(\frac{l_{2}}{2} \cos \varphi \sin \theta \dot{\mathbf{i}}_{0}+\frac{l_{2}}{2} \cos \varphi \cos \theta \mathbf{j}_{0}-\left(\frac{l_{2}}{2} \sin \varphi+l_{1}\right) \mathbf{k}_{0}\right) \\
& =\frac{l_{2}}{2}(\dot{\theta} \cos \varphi \cos \theta-\dot{\varphi} \cos \varphi \sin \theta) \mathbf{i}_{0} \\
& -\frac{l_{2}}{2}(\dot{\theta} \cos \varphi \sin \theta+\dot{\varphi} \sin \varphi \cos \theta) \mathbf{j}_{0}-\frac{l_{2}}{2} \dot{\varphi} \cos \varphi \mathbf{k}_{0} \\
\mathbf{v}_{C_{3}} & =\frac{d}{d t} \mathbf{r}_{C_{3}} \\
& =\frac{d}{d t}\left(l_{C_{3}} \cos \varphi \sin \theta \mathbf{i}_{0}+l_{C_{3}} \cos \varphi \cos \theta \mathbf{j}_{0}-\left(l_{C_{3}} \sin \varphi+l_{1}\right) \mathbf{k}_{0}\right) \\
& =\left[\dot{l}_{C_{3}} \cos \varphi \sin \theta+l_{C_{3}} \dot{\theta} \cos \varphi \cos \theta-l_{C_{3}} \dot{\varphi} \cos \varphi \sin \theta\right] \mathbf{i}_{0} \\
& +\left[i_{C_{3}} \cos \varphi \cos \theta-l_{C_{3}} \dot{\theta} \cos \varphi \sin \theta-l_{C_{3}} \dot{\varphi} \sin \varphi \cos \theta\right] \mathbf{j}_{0} \\
& -\left[\dot{l}_{C_{3}} \sin \varphi+l_{C_{3}} \dot{\varphi} \cos \varphi\right] \mathbf{k}_{0}
\end{aligned}
$$

The velocity of $C_{3}$ in (0) can also be computed (if needed) with respect to the reference frame (2) by

$$
\mathbf{v}_{C_{3}}=\frac{d}{d t} \mathbf{r}_{C_{3}}=\frac{{ }^{(2)} d}{d t} \mathbf{r}_{C_{3}}+\boldsymbol{\omega}_{20} \times \mathbf{r}_{C_{3}},
$$


The linear accelerations of the mass centers $C_{1}, C_{2}$, and $C_{3}$ are calculated using

$$
\begin{aligned}
& \mathbf{a}_{C_{1}}=\frac{d}{d t} \mathbf{v}_{C_{1}}=\frac{{ }^{(1)} d}{d t} \mathbf{v}_{C_{1}}+\boldsymbol{\omega}_{10} \times \mathbf{v}_{C_{1}} \\
& \mathbf{a}_{C_{2}}=\frac{d}{d t} \mathbf{v}_{C_{2}}=\frac{{ }^{(1)} d}{d t} \mathbf{v}_{C_{2}}+\boldsymbol{\omega}_{20} \times \mathbf{v}_{C_{2}} \\
& \mathbf{a}_{C_{3}}=\frac{d}{d t} \mathbf{v}_{C_{3}}=\frac{{ }^{(2)} d}{d t} \mathbf{v}_{C_{3}}+\boldsymbol{\omega}_{20} \times \mathbf{v}_{C_{3}} .
\end{aligned}
$$

\section{Lagrange's Equations}

The dynamical equations governing the manipulator arm can be written as in 25] using Lagrange's equations

$$
\frac{d}{d t}\left(\frac{\partial T}{\partial \dot{q}_{j}}\right)-\frac{\partial T}{\partial q_{j}}=Q_{j}, \quad j=1,2,3
$$

where $Q_{j}$ are the generalized forces, $q_{1}=\theta, q_{2}=\varphi$ and $q_{3}=l_{C_{3}}$ are the time dependent generalized coordinates, and $T$ is the kinetic energy of the system. The total kinetic energy of the manipulator can be expressed as

$$
T=\sum_{j=1}^{3} T_{r}=T_{1}+T_{2}+T_{3},
$$

The kinetic energies $T_{1}, T_{2}$ and $T_{3}$ of the links 1,2 and 3 are

$$
T_{j}=\frac{1}{2} m_{j} \mathbf{v}_{C_{j}} \cdot \mathbf{v}_{C_{j}}+\frac{1}{2} \boldsymbol{\omega}_{j 0} \cdot\left(\bar{I}_{j} \cdot \boldsymbol{\omega}_{j 0}\right), \quad j=1,2,3
$$

where $m_{1}, m_{3}$ and $m_{3}$ are the masses and $I_{1}, I_{2}$ and $I_{3}$ are the central inertia dyadics of the links 1, 2 and 3, expressed as

$$
I_{j}=\left(I_{j x} \mathbf{1}_{j}\right) \mathbf{l}_{j}+\left(I_{j y} \mathbf{J}_{j}\right) \mathbf{j}_{j}+\left(I_{j z} \mathbf{k}_{j}\right) \mathbf{k}_{j}, \quad j=1,2,3
$$

The generalized forces $Q_{r}$ are given as in [25] by

$$
Q_{j}=\frac{\partial \boldsymbol{\omega}}{\partial \dot{q}_{j}} \cdot \mathbf{T}+\frac{\partial \mathbf{v}_{P}}{\partial \dot{q}_{j}} \cdot \mathbf{R}, \quad j=1,2, \ldots,
$$

where $\mathbf{T}$ is the equivalent acting couple or torque, $\mathbf{R}$ is the applied force at the point $P, \boldsymbol{\omega}$ is the angular velocity expressed in the reference frame in $(0), \mathbf{v}_{P}$ is the velocity of the point $P$ in the reference frame (0). For the manipulator shown 
in Fig. 1, the generalized forces $Q_{1}, Q_{2}$, and $Q_{3}$ are made by the gravitational and contact forces that drive the links 1,2, and 3. The set of contact forces transmitted from link 0 to link 1 , and from link 1 to link 2 can be replaced by the torques $\mathbf{T}_{01}$ and $\mathbf{T}_{12}$. The set of contact forces exerted by link 2 on link 3 can be replaced by a force $\mathbf{F}_{23}$. The expressions $\mathbf{T}_{01}, \mathbf{T}_{12}$, and $\mathbf{F}_{23}$ are

$$
\begin{aligned}
& \mathbf{T}_{01}=T_{01 x} \mathbf{l}_{1}+T_{01 y} \mathbf{j}_{1}+T_{01 z} \mathbf{k}_{1} \\
& \mathbf{T}_{12}=T_{12 x} \mathbf{l}_{2}+T_{12 y} \mathbf{j}_{2}+T_{12 z} \mathbf{k}_{2} \\
& \mathbf{F}_{23}=F_{23 x} \mathbf{l}_{2}+F_{23 y} \mathbf{j}_{2}+F_{23 z} \mathbf{k}_{2} .
\end{aligned}
$$

One can express the contribution $\left(Q_{j}\right)_{1},\left(Q_{j}\right)_{2}$ and $\left(Q_{j}\right)_{3}$ to the generalized active force $Q_{j}$ (for any $j=1,2,3$ ) of all the forces and torques acting on the links 1,2 and 3 as in [25] by

$$
\begin{aligned}
\left(Q_{j}\right)_{1} & =\frac{\partial \boldsymbol{\omega}_{10}}{\partial \dot{q}_{j}} \cdot\left(\mathbf{T}_{01}-\mathbf{T}_{12}\right)+\frac{\partial \mathbf{v}_{C_{1}}}{\partial \dot{q}_{j}} \cdot \mathbf{G}_{1} \\
\left(Q_{j}\right)_{2} & =\frac{\partial \boldsymbol{\omega}_{20}}{\partial \dot{q}_{j}} \cdot \mathbf{T}_{12}+\frac{\partial \mathbf{v}_{C_{2}}}{\partial \dot{q}_{j}} \cdot \mathbf{G}_{2}+\frac{\partial \mathbf{v}_{C_{32}}}{\partial \dot{q}_{j}} \cdot\left(-\mathbf{F}_{23}\right), \\
\left(Q_{j}\right)_{3} & =\frac{\partial \mathbf{v}_{C_{3}}}{\partial \dot{q}_{j}} \cdot \mathbf{G}_{3}+\frac{\partial \mathbf{v}_{C_{3}}}{\partial \dot{q}_{j}} \cdot \mathbf{F}_{23},
\end{aligned}
$$

where $\mathbf{v}_{C_{32}}=\mathbf{v}_{C_{3}}-i_{C_{3}} \mathbf{i}_{2}$, and the gravitational forces $\mathbf{G}_{1}, \mathbf{G}_{2}, \mathbf{G}_{3}$ exerted on the links 1, 2, and 3 can be calculated using $\mathbf{G}_{j}=-m_{j} g \mathbf{k}_{0}$.

From Eq. 23 one can calculate the generalized active force $Q_{1}, Q_{2}$ and $Q_{3}$ of all the forces and torques acting on the links 1, 2, and 3 by

$$
\begin{aligned}
& Q_{r}=\left(Q_{r}\right)_{1}+\left(Q_{r}\right)_{2}+\left(Q_{r}\right)_{3} \\
& =\frac{\partial \boldsymbol{\omega}_{10}}{\partial \dot{q}_{j}} \cdot\left(\mathbf{T}_{01}-\mathbf{T}_{12}\right)+\frac{\partial \mathbf{v}_{C_{1}}}{\partial \dot{q}_{j}} \cdot \mathbf{G}_{1}+\frac{\partial \boldsymbol{\omega}_{20}}{\partial \dot{q}_{r}} \cdot \mathbf{T}_{12}+\frac{\partial \mathbf{v}_{C_{2}}}{\partial \dot{q}_{j}} \cdot \mathbf{G}_{2}+\frac{\partial \mathbf{v}_{C_{32}}}{\partial \dot{q}_{r}} \cdot\left(-\mathbf{F}_{23}\right) \\
& +\frac{\partial \mathbf{v}_{C_{3}}}{\partial \dot{q}_{j}} \cdot \mathbf{G}_{3}+\frac{\partial \mathbf{v}_{C_{3}}}{\partial \dot{q}_{j}} \cdot \mathbf{F}_{23}, \quad j=1,2,3 .
\end{aligned}
$$

\section{Results}

To illustrate trajectory generation a numerical example (using Matlab 25]) is presented for a robotic manipulator composed link 1 of maximal height $l_{1}=0.05$ $\mathrm{m}$, link 2 of length $l_{2}=0.65 \mathrm{~m}$ and sliding link 3 of length $l_{3}=0.65 \mathrm{~m}$. The 


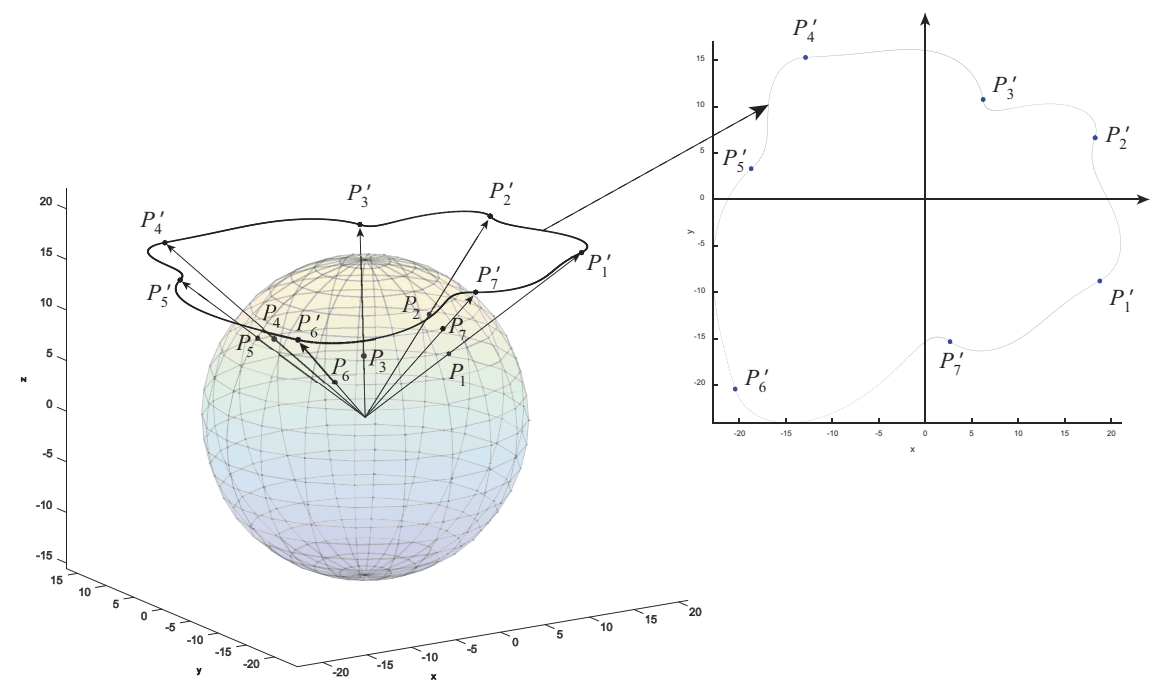

Figure 5: Gnomic projection of the via points and associated polar interpolation

rigid guide and sliding link are rotating with an angular velocity $\omega=1 \mathrm{rad} / \mathrm{s}$ about the $z$-guide. The radial distance related to the manipulator length, and the polar and azimuthal angles of the manipulator via-points are shown in Table 1 .

Table 1: Via-points coordinates the manipulator arm should follow

\begin{tabular}{ccccccccc}
\hline \hline Variable Name & \multicolumn{1}{c}{ Value } \\
\hline$i$ & 1 & 2 & 3 & 4 & 5 & 6 & 7 & $8=1$ \\
$\theta_{i}$ & 20 & 60 & 130 & 170 & 225 & 280 & 335 & $360+20$ \\
$\varphi_{i}$ & 39 & 51 & 37 & 38 & 28 & 45 & 36 & 39 \\
$l_{P_{i}}$ & 10 & 6.5 & 9 & 11 & 13 & 11 & 8 & 10 \\
\hline
\end{tabular}

The 3D end-effector trajectory for the manipulator configuration in Table 1 is shown in Fig. 6 (red curve). The trajectory shown in 5 represents the polar trajectory of the end-effector obtained on the gnomic projective plane using polar interpolation curves expressed as a Hermite-type functions. The plot showing a top view of the trajectory of the sequence of the projected via- 


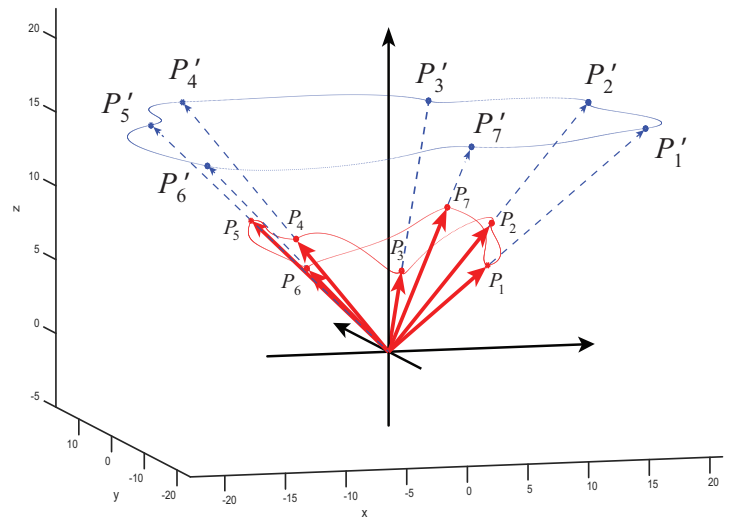

Figure 6: Manipulator trajectory along the via points (red curve) and gnomic trajectory (blue curve)

points is also presented in Fig. 5. The plane in which the radial trajectory of the projected via-points is developed is tangent to the sphere of radius $l_{\text {Max }}=$ $\max \left\{l_{P_{i}}\right\}_{i=\overline{0, N_{i}}}$. The arrows pointing toward the projected points represent the position vectors towards the gnomic via-points.

The manipulator trajectory shown in Fig. 6 (red curve) is obtained through the combination of the inverse geometric transformation applied to the polar piecewise interpolants that approximate the gnomic polar trajectories (left and right motion) and the azimuthal trajectory (up and down motion) generated by polar piecewise interpolants. The red arrows pointing toward the via-points represent the position vectors related to the via-points in the spherical coordinates system.

The computation also shows that the manipulator arm is inside the working envelope/workspace 4, that is, the end-effector of the manipulator arm can reach all the via-points in Table 1 along the planned trajectory shown in Fig. 6 This emphasize the importance of nodal via points as possible extreme points of the reachable workspace (working volume) of the prescribed curve. The smoothness of the trajectory proves the effectiveness of the approach, and as a result, the axial force acting on the manipulator along the motion is also continuous. Since the trajectory is not dependent on the manipulator arm 
geometry and can be expressed as a combination of rotations about a fixed point (manipulator base), the actual model can be used in path planning of any kind of manipulators. During path planning, the use of polar and/or Cartesian piecewise interpolating curves $[8,23$ ] can be related with the robot dynamics $[8,18,25]$ through its geometry [8], therefore, the obtained trajectory is accurate, efficient and smooth, an excellent guarantee of a good kinematics/dynamics performance.

\section{Conclusions}

In this paper the modelling and 3D trajectory planning of an extensible rotating manipulator is considered. Polar zenithal gnomic perspective projections of the $3 \mathrm{D}$ via points and polar piecewise interpolants are considered for an initial planar trajectory generations. The 3D polar trajectories are generated using the inverse geometric transformation applied to the polar piecewise interpolants that approximate the planar gnomic trajectory of the $3 \mathrm{D}$ via points. The related azimuthal trajectory is related to the 3D polar trajectories by polar piecewise interpolants on the azimuthal angles. Trajectory smoothness obtained through the use of piecewise interpolants with continuous derivatives relate with the robot dynamics through its geometry, resulting in a accurate, efficient and smooth trajectory well suited to computer implementation. A numerical example is presented to illustrate trajectory planning and verify the proposed approach.

\section{References}

[1] Adhami L, Coste E. Optimal planning for minimally invasive surgical robots. IEEE Transactions on Robotics and Automation 2003; 19(5):854863.

[2] Le Boudec B, Saad M, Negruizian V. Modeling and adaptive control of redundant robots. Math. Comp. Sim. 2006; 19:395-403. 
[3] Bobrow JE, Dubowsky S, Gibson JS. Time-optimal control of robotic manipulators along specified paths. International Journal of Robotics Research $1985 ; 4(3): 3-17$.

[4] Cao Y, Lu K, Li X, Zang Y. Accurate numerical methods for computing 2D and 3D robot workspace. International Journal of Advanced Robotic Systems 2011; 8(6):1-13.

[5] Chapra SC, Canale RP. Numerical Methods for Engineers. McGraw-Hill, 6th Ed., 2010.

[6] Constantinescu D, Croft EA. Smooth and time-optimal trajectory planning for industrial manipulators along specified paths. Journal of Robotic Systems 2000; 17(5):233-249.

[7] Dong J, Ferreira PM, Stori JA. Feed-rate optimization with jerk constraints for generating minimum-time trajectories. Int. J. Mach. Tools Manuf. 2007; 47(12):1941-1955.

[8] Dupac M. Smooth trajectory generation for rotating extensible manipulators. Mathematical Methods in the Applied Sciences http://onlinelibrary.wiley.com/doi/10.1002/mma.4210/full, 2016.

[9] Dupac M. 3D trajectory generation for rotating extensible manipulators using zenithal gnomic projection and polar piecewise interpolants. Proceedings of the 17th International Conference on Computational and Mathematical Methods in Science and Engineering 2017; 2121-2129.

[10] Garcia EAM. Numerical modeling in Robotics, Omnia Science, 2015.

[11] Gariblu H, Korayem MH. Trajectory Optimization of Flexible Mobile Manipulators. Robotica 2006; 24(03):333-335.

[12] Gasparetto A, Boscariol P, Lanzutti A, Vidoni R. Trajectory planning in Robotics. Mathematics in Computer Science 2012; 6:269-279. 
[13] Goodman TNT, Lee SL. B-splines on the Circle and Trigonometric Bsplines. Approximation Theory and Spline Functions 1984; 136:297-325.

[14] Gross M, Staniforth A. Cubic-spline interpolation on a non-uniform latitude-longitude grid: achieving cross- and circum-polar continuity. Atmos. Sci. Let. 2010; 11:229-23.

[15] Hsu D, Kindel R, Latombe JC, Rock S. Randomized kinodynamic motion planning with moving obstacles. Int. J. Robot. Res. 2002; 21(3):233-255.

[16] Iwashita Y. Piecewise Polynomial interpolation. OpenGamma Quantitative Research 2014; 15:1-22.

[17] Kang IG, Park FC. Cubic Spline Algorithms for Orientation Interpolation. International Journal for Numerical Methods in Engineering 1999; 46:4564.

[18] Kalyoncu M. Mathematical modelling and dynamic response of a multistraight-line path tracing flexible robot manipulator with rotatingprismatic joint. Applied Mathematical Modelling 2008; 32:1087-1098.

[19] Korayem MH, Nikoobin A, Azimi V. Maximum Load Carrying Capacity of Mobile Manipulators: Optimal Control Approach. Robotica 2009; $27(1): 147-159$.

[20] Korayem MH, Nikoobin A. Maximum-Payload Path Planning for Redundant Manipulator Using Indirect Solution of Optimal Control Problem. International Journal of Advanced Manufacturing Technology 2009; 44(7$8): 725-736$.

[21] Korayem MH, Azimirad V, Vatanjou H, Korayem AH. Maximum load determination of nonholonomic mobile manipulator using hierarchical optimal Control. Robotica 2012; 30(1):53-65.

[22] Kumar V, Zefran M, Ostrowski JP. Motion Planning and Control of Robots. Handbook of Industrial Robotics, New-York, 2007. 
[23] Lavery JE. Shape-preserving, multiscale interpolation by univariate curvature-based cubic L1 splines in Cartesian and polar coordinates. Computer Aided Geometric Design 2002; 19:257-273.

[24] Lin CS, Chang PR, Luh JYS. Formulation and optimization of cubic polynomial joint trajectories for industrial robots. IEEE Trans. Autom. Control 1983; 28(12):1066-1073.

[25] Marghitu DB, Dupac M. Advanced Dynamics: Analytical and Numerical Calculations with Matlab. Springer: New York, 2012.

[26] Murray RM, Li Z, Sastry SS. A Mathematical Introduction to Robotic Manipulation. CRC Press: Boca Raton, 1994.

[27] du Plessis LJ, Snyman JA. Trajectory-planning through interpolation by overlapping cubic arcs and cubic splines, International Journal for Numerical Methods in Engineering, 2003; 57:1615-1641.

[28] Sanchez-Reyes J. Single-valued spline curves in polar coordinates. Computer Aided Design 1992; 24:307-315.

[29] $\mathrm{Su} \mathrm{B}$, Zou L. Manipulator trajectory planning based on the algebraic trigonometric Hermite blended interpolation spline. Procedia Engineering 2012; 29:2093-2097.

[30] Wang C-WE. Dynamic Motion Planning For Robot Manipulators Using B-Splines. PhD Dissertation, University of California, Irvine, 2001.

[31] Yo D-Y. Numerical Analysis of Workspaces of Multibody Mechanical Systems. PhD Thesys, University of Iowa, Iowa, 1988. 